\title{
Plasma chemokine CXC motif-ligand 16 as a predictor of renal prognosis in immunoglobulin A nephropathy
}

\author{
Ran Luo, Yi Yang, Yi-Chun Cheng, Dan Chang, Ting-Ting Liu, Yue-Qiang Li, Wei Dai, Mei-Ying Zuo, \\ Yu-Lin Xu, Chun-Xiu Zhang, Shu-Wang Ge, Gang Xu \\ Department of Nephrology, Division of Internal Medicine, Tongji Hospital, Tongji Medical College, Huazhong University of Science and \\ Technology, Wuhan 430030, China \\ Contributions: (I) Conception and design: SW Ge; (II) Administrative support: G Xu; (III) Provision of study materials or patients: R Luo; (IV) \\ Collection and assembly of data: R Luo; (V) Data analysis and interpretation: R Luo; (VI) Manuscript writing: All authors; (VII) Final approval of \\ manuscript: All authors. \\ Correspondence to: Shu-Wang Ge, PhD. Department of Nephrology, Division of Internal Medicine, Tongji Hospital, Tongji Medical College, \\ Huazhong University of Science and Technology, Wuhan 430030, China. Email: geshuwang@tjh.tjmu.edu.cn; Gang Xu, PhD. Department of \\ Nephrology, Division of Internal Medicine, Tongji Hospital, Tongji Medical College, Huazhong University of Science and Technology, Wuhan \\ 430030, China. Email: xugang@tjh.tjmu.edu.cn.
}

\begin{abstract}
Background: There are few non-invasive biomarkers that have been identified to improve the risk stratification of patients with IgA nephropathy (IgAN). CXCL16 has been shown to play a key role as a chemoattractant, adhesion, and fibrosis factor in inflammatory disease. This study evaluated the potential for CXCL16 plasma as a potential biomarker in patients with IgAN.

Methods: Plasma CXCL16 was measured in 230 patients with renal biopsied IgAN enrolled from 2012 to 2014. The patients were followed for 41.3 months, with a $50 \%$ reduction in estimated glomerular filtration rate or end-stage renal disease as endpoints.

Results: The plasma CXCL16 levels in IgAN patients were strongly correlated with the uric acid, estimated glomerular filtration rate and tubular atrophy/interstitial fibrosis score in multivariate analysis. Furthermore, counts of $\mathrm{CD}^{+} \mathrm{T}$ cells, $\mathrm{CD}^{+} \mathrm{T}$ cells, and $\mathrm{CD} 20^{+} \mathrm{B}$ cells in renal biopsies of IgAN patients were significantly correlated with the plasma CXCL16 levels, but not CD68 $8^{+}$macrophage. Lastly, we concluded that patients with higher levels of plasma CXCL16 had an increased risk of poor renal outcome compared to those with lower levels. There was no association between the polymorphisms and clinical parameters of CXCL16, including the levels and prognosis of plasma CXCL16.

Conclusions: Plasma CXCL16 levels were associated with clinical parameters; pathological damage; $\mathrm{CD}^{+} \mathrm{T}$ cell, $\mathrm{CD}^{+} \mathrm{T}$ cell, and $\mathrm{CD} 20^{+} \mathrm{B}$ cell infiltration in renal tissue; and renal outcome in IgAN patients. Plasma CXCL16 might be a potential prognosis predictor in Chinese IgAN patients.
\end{abstract}

Keywords: CXCL16; IgA nephropathy (IgAN); inflammation cells; prognosis; polymorphisms

Submitted Nov 20, 2019. Accepted for publication Jan 18, 2020.

doi: 10.21037/atm.2020.02.05

View this article at: http://dx.doi.org/10.21037/atm.2020.02.05

\section{Introduction}

IgA nephropathy (IgAN) is the most common type of primary glomerulonephritis worldwide (1). Approximately $30 \%$ of patients eventually develop end-stage renal disease (ESRD) within 20-30 years after renal biopsy $(1,2)$. Unfortunately, IgAN diagnosis is based on kidney biopsy, and there is no approved IgAN progression diagnostic blood biomarker. Biopsy specimens that meet IgAN diagnostic criteria have a wide range of pathological changes that are often reflected in IgAN patients with variable clinical prognosis. Given the invasive nature, there is urgent need for clinical practice for non-invasive biomarkers associated 
with IgAN prognosis.

Another common pathological characteristic associated with IgAN is renal interstitial inflammatory infiltration. Previous studies have reported several inflammatory cell infiltration with prognostic significance in chronic kidney diseases (3-5). Our team also demonstrated that the accumulation of interstitial $\mathrm{CD}^{+} \mathrm{T}$ cells, $\mathrm{CD}^{+} \mathrm{T}$ cells, $\mathrm{CD} 20^{+} \mathrm{B}$ cells, $\mathrm{CD} 68^{+}$macrophage or dendritic cells, were correlated with decreased renal function and poor renal outcomes $(6,7)$. The migration of leukocytes depends on a variety of adhesion molecules and chemokines (8). Interestingly, the chemokine CXC motif-ligand 16 (CXCL16) was found to play an important role in the development of inflammatory diseases (9).

CXCL16 is a chemokine belonging to the CXC chemokine family (10), which acts as a classical chemoattractant for CXCR6-expressing cells, including $\mathrm{CD}^{+} \mathrm{T}$ cells, $\mathrm{CD}^{+} \mathrm{T}$ cells, NK cells, invariant $\mathrm{NKT}$ cells, plasma cells, and monocytes (11-13). CXCL16 serves as an important pathogenic mediator in inflammatory diseases $(9,14,15)$. Several polymorphic variants of CXCL16 were associated with the risk of various diseases, including coronary artery disease (16) and inflammatory bowel disease (17).

In addition, in recent studies CXCL16 has been reported to be associated with renal diseases. Serum and urinary CXCL16 was higher in lupus nephritis, particularly in periods of disease activity (15). In diabetic kidney disease, plasma CXCL16 levels were found to be independently correlated with estimated glomerular filtration rate (eGFR) and serum albumin $(18,19)$. In fact, blocking the activities of CXCL16 has been to shown to prevent the progression of anti-GBM glomerulonephritis even when the disorder is found (20). These findings suggest that CXCL16 may be a marker of renal disease. Whereas prolific data have been published on clinical data, little is known about CXCL16's associations with renal pathology and clinic outcomes. Larger cohort studies with a long follow-up period to evaluate the predictive value of the chemokine CXCL16 in renal inflammation and outcomes are needed.

The purpose of this study was to investigate plasma CXCL16 characteristics in patients with IgAN. We investigated plasma levels of CXCL16 at time of renal biopsy and analyzed its associations with clinical and pathological characteristics; the density of $\mathrm{CD}^{+} \mathrm{T}$ cells, $\mathrm{CD}^{+} \mathrm{T}$ cells, $\mathrm{CD} 20^{+} \mathrm{B}$ cells, and $\mathrm{CD}^{+} 8^{+}$macrophages; and renal CXCL16 expression and outcome by using a large IgAN cohort of Chinese patients.

\section{Methods}

\section{Study cohort}

In this study, all-adult ( $\geq 18$ years) patients with biopsyproven primary IgAN from January 2012 to January 2014 at Tongji Hospital of Huazhong University of Science and Technology were enrolled $(n=230)$. The patients were followed up until April 2019 for an average of 41.3 months. Patients with Henoch-Schönlein purpura, lupus nephritis, rheumatoid arthritis, chronic liver disease, diabetes, Crohn's disease, cancers, cardiovascular diseases or whose renal biopsy specimen contained less than 8 glomeruli, were excluded from the study. The renal outcomes were the survival from a $50 \%$ reduction in eGFR or ESRD (eGFR $<15 \mathrm{~mL} / \mathrm{min}$ per $1.73 \mathrm{~m}^{2}$ ).

This study conformed to the Ethical Committee of Huazhong University of Science and Technology guidelines (No. TJ-IRB20181108). All procedures conducted in this study followed the institutional and/or national research committee's ethical standards and the Helsinki Declaration of 1964 and its later amendments or comparable ethical standards. All participants included in the study gave informed consent.

\section{Plasma CXCL16 measurement}

CXCL16 levels were measured in plasma samples which were obtained from all IgAN patients on the day of renal biopsy by using a Quantikine CXCL16 (R\&D Systems, Minneapolis, MN, USA) ELISA kit following the manufacturer's instruction.

Receiver-operating characteristic (ROC) curves showed the optimal cut-off for plasma CXCL16 levels to predict the renal outcomes endpoint was $3.016 \mathrm{ng} / \mathrm{mL}$ [area under the curve (AUC) 0.675 , specificity $66 \%$, sensitivity $65 \%$, $\mathrm{P}=0.016]$. Patients with plasma CXCL16 levels higher than the cut-off value were defined as the high CXCL16 group, and patients with plasma CXCL16 levels lower than the cut-off value were defined as the low CXCL16 group.

\section{Immunobistochemistry}

Immunohistochemistry (IHC) was performed as described previously in paraffin-embedded tissue sections at a thickness of $4 \mathrm{~mm}$ (21). For human biopsy samples, CD4, CD20, CD68 (Gene Tech, Shanghai, China), CD8 (Maxim-bio, Fuzhou, China), and CXCL16 (Peprotech, Rocky Hill, NJ, USA) antibody were used. Quantitative evaluation of inflammatory 
Table 1 The demographic and clinical parameters of the patients with $\operatorname{IgAN}(\mathrm{N}=230)$

\begin{tabular}{lc}
\hline Parameters & Results \\
\hline Age (years; mean \pm SD) & $34.5 \pm 9.7$ \\
Gender (female/male) & $139 / 91$ \\
MAP (mmHg; mean \pm SD) & $98.6 \pm 14.9$ \\
Hypertension, $n(\%)$ & $93(40.4)$ \\
Microscopic hematuria, $n(\%)$ & $221(96.1)$ \\
Gross hematuria, $n(\%)$ & $32(13.9)$ \\
Albumin (g/L; mean \pm SD) & $39.3 \pm 5.3$ \\
Serum creatinine $(\mu$ mol/L; median, IQR) & $80.0,63.0-109.8$ \\
eGFR (mL/min per 1.73 m ${ }^{2} ;$ median, IQR) & $93.2,63.3-116.6$ \\
$24-h$ urine protein (g/d; median, IQR) & $0.84,0.40-1.74$ \\
CXCL16 (ng/mL; mean \pm SD) & $2.89 \pm 0.82$ \\
\hline
\end{tabular}

Values are expressed as mean \pm standard deviation, median (25th percentile-75th percentile), or number (percentage). MAP, mean arterial pressure; eGFR, estimated glomerular filtration rate; IQR, interquartile range. eGFR was calculated with the CKD-EPI equation. 24-h urine protein was missing in $10 \%$ of cases.

cells was counted under 5 equivalent high-power cortical fields (HPFs) $(\times 400)$ and was expressed as the average number of cells per high-power cortical fields by Image-Pro-Plus software6.0 (Media Cybernetics, Silver Spring, MD, USA).

\section{Statistical analyses}

The statistical analyses were carried out using SPSS 23.0 software (SPSS, Chicago, IL, USA) and GraphPad Prism version 6 software (Graph software, San Diego, CA, USA). Correlations were evaluated using nonparametric Spearman's and parametric Pearson's correlation test. Multivariate logistic regression analysis was performed to identify the independent relationship between plasma CXCL16 levels and clinical parameters. We used Cox regression and Kaplan-Meier survival curves to analyze the association between plasma CXCL16 levels and renal outcome, and the significance was determined by the logrank test. The optimal cut-off value for high and low expression for CXCL16 was obtained by ROC curve analysis. All of the statistical analyses were two-tailed. $\mathrm{P}<0.05$ was considered statistically significant.

\section{Results}

\section{Cobort description}

The 230 patients had an initial eGFR of 93.2 (63.3-
116.6) $\mathrm{mL} / \mathrm{min}$ per $1.73 \mathrm{~m}^{2}$. The cohort was $39.5 \%$ male (Table 1). Initial proteinuria was $0.84(0.40-1.74) \mathrm{g} / \mathrm{d}$, and mean arterial pressure (MAP) was $98.6 \pm 14.9 \mathrm{mmHg}$. Out of 230 patients, gross hematuria at onset was present in $32(14.0 \%)$ patients and hypertension in $93(40.4 \%)$ patients. Patients were followed for a median of 41.3 (28.1-50.0) years.

\section{Correlations between CXCL16 levels and clinical parameters of the IgAN patients}

We evaluated CXCL16 in plasma of all IgAN patients at the time of renal biopsy. The plasma level of CXCL16 was $2.89 \pm 0.82 \mathrm{ng} / \mathrm{mL}$. There was a significantly inverse correlation between plasma CXCL16 levels and eGFR, and albumin $(\mathrm{r}=-0.411, \mathrm{P}<0.001, \mathrm{r}=-0.218, \mathrm{P}=0.001$; Figure 1$)$, and a positive correlation between plasma CXCL16 and Scr, and 24-h urine protein $(\mathrm{r}=0.367, \mathrm{P}<0.001, \mathrm{r}$ $=0.232, \mathrm{P}=0.001$ respectively; Figure $1 A, D)$. Furthermore, plasma CXCL16 levels were significantly correlated with other clinical data, including blood urea nitrogen, uric acid, serum $\mathrm{K}$, serum $\mathrm{P}$, and serum $\mathrm{Ca}$ (Table 2). In multivariate linear regression analysis eGFR $(\beta$ $=-0.008, \mathrm{P}=0.018)$ and uric acid $(\beta=0.002, \mathrm{P}=0.013)$ were independently associated with plasma CXCL16 (Table 2). These results suggest that plasma CXCL16 levels were correlated with IgAN renal function. 

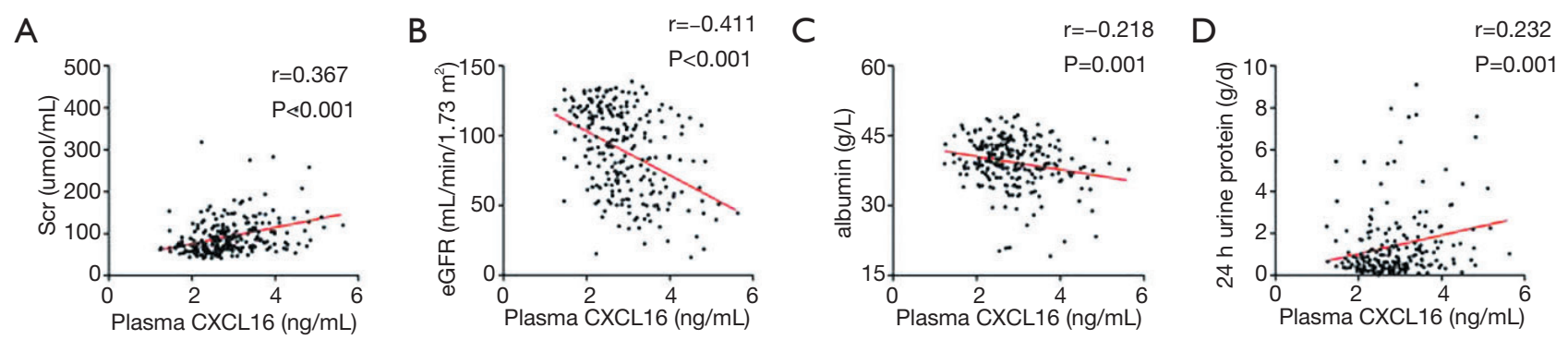

Figure 1 The correlations between the plasma CXCL16 levels and Scr (A), eGFR (B), albumin (C), and 24-h urine protein (D) in univariate analysis.

Table 2 Correlations between the levels of plasma CXCL16 and other clinical parameters in the patients with IgAN in univariate analysis and multivariate analysis $(\mathrm{N}=230)$

\begin{tabular}{|c|c|c|c|c|}
\hline Parameters & \multicolumn{2}{|c|}{ Univariate linear regression } & \multicolumn{2}{|c|}{ Multivariate linear regression } \\
\hline ALB $(g / L)$ & -0.218 & 0.001 & -0.020 & 0.200 \\
\hline $\operatorname{Scr}(\mu \mathrm{mol} / \mathrm{L})$ & 0.367 & $<0.001$ & -0.004 & 0.099 \\
\hline UA (mmol/L) & 0.277 & $<0.001$ & 0.002 & 0.013 \\
\hline eGFR $\left(\mathrm{mL} / \mathrm{min}\right.$ per $\left.1.73 \mathrm{~m}^{2}\right)$ & -0.411 & $<0.001$ & -0.008 & 0.018 \\
\hline 24-h urine protein $(\mathrm{g} / \mathrm{d})$ & 0.232 & 0.001 & 0.015 & 0.669 \\
\hline $\mathrm{K}(\mathrm{mmol} / \mathrm{L})$ & 0.209 & 0.002 & 0.133 & 0.445 \\
\hline $\mathrm{Ca}(\mathrm{mmol} / \mathrm{L})$ & -0.144 & 0.031 & -0.187 & 0.687 \\
\hline
\end{tabular}

eGFR was calculated with the CKD-EPI equation. 24-h urine protein was missing in $10 \%$ of cases.

\section{Associations between CXCL16 levels in our IgAN patient cobort and the MEST-C pathological scores}

We then analyzed the association between plasma CXCL16 levels and the Oxford Classification. By univariate analysis, we found that patients with mesangial hypercellularity had higher plasma CXCL16 levels $(\mathrm{P}<0.001)$. Plasma CXCL16 levels increased with tubular atrophy/interstitial fibrosis score $(\mathrm{P}<0.001)$. Patients with a renal crescent proportion of more than $25 \%$ had higher plasma CXCL16 levels $(\mathrm{P}=0.018)$. Comparable levels of plasma CXCL16 were present in patients without or with endocapillary hypercellularity $(\mathrm{P}=0.638)$ and patients absent or present of segmental glomerulosclerosis $(\mathrm{P}=0.313)$.

Furthermore, with multivariate linear regression analysis of the 4 pathology variables and the clinical data set (age, gender, MAP and 24-h urine protein), we found that the plasma CXCL16 levels were still significantly correlated with the tubular atrophy/interstitial fibrosis score $(\mathrm{P}=0.023)$ and nearly correlated with the crescent proportion $(\mathrm{P}=0.070)$, whereas association with other pathological variables failed to reach independent statistical significance in the multivariate analysis model. The details of this analysis are listed in Table 3. These results demonstrate that renal pathology was correlated with plasma CXCL16 levels, especially tubular atrophy/interstitial fibrosis score.

\section{Expression of renal tissue CXCL16 and correlation with plasma CXCL16 levels}

Renal CXCL16 was expressed in cytoplasm and a part of the membrane. We calculated the CXCL16 positive renal tube per renal tissue area for the quantification analysis of renal CXCL16 expression with plasma CXCL16 levels. We found predominantly positive correlations between the renal CXCL16 expression and plasma CXCL16 levels $(\mathrm{r}=0.316, \mathrm{P}=0.018$; Figure 2). These findings suggest that 
Table 3 Correlations between plasma CXCL16 levels and the Oxford Classification in IgAN patients: univariate and multivariate linear regression analysis $(\mathrm{N}=230)$

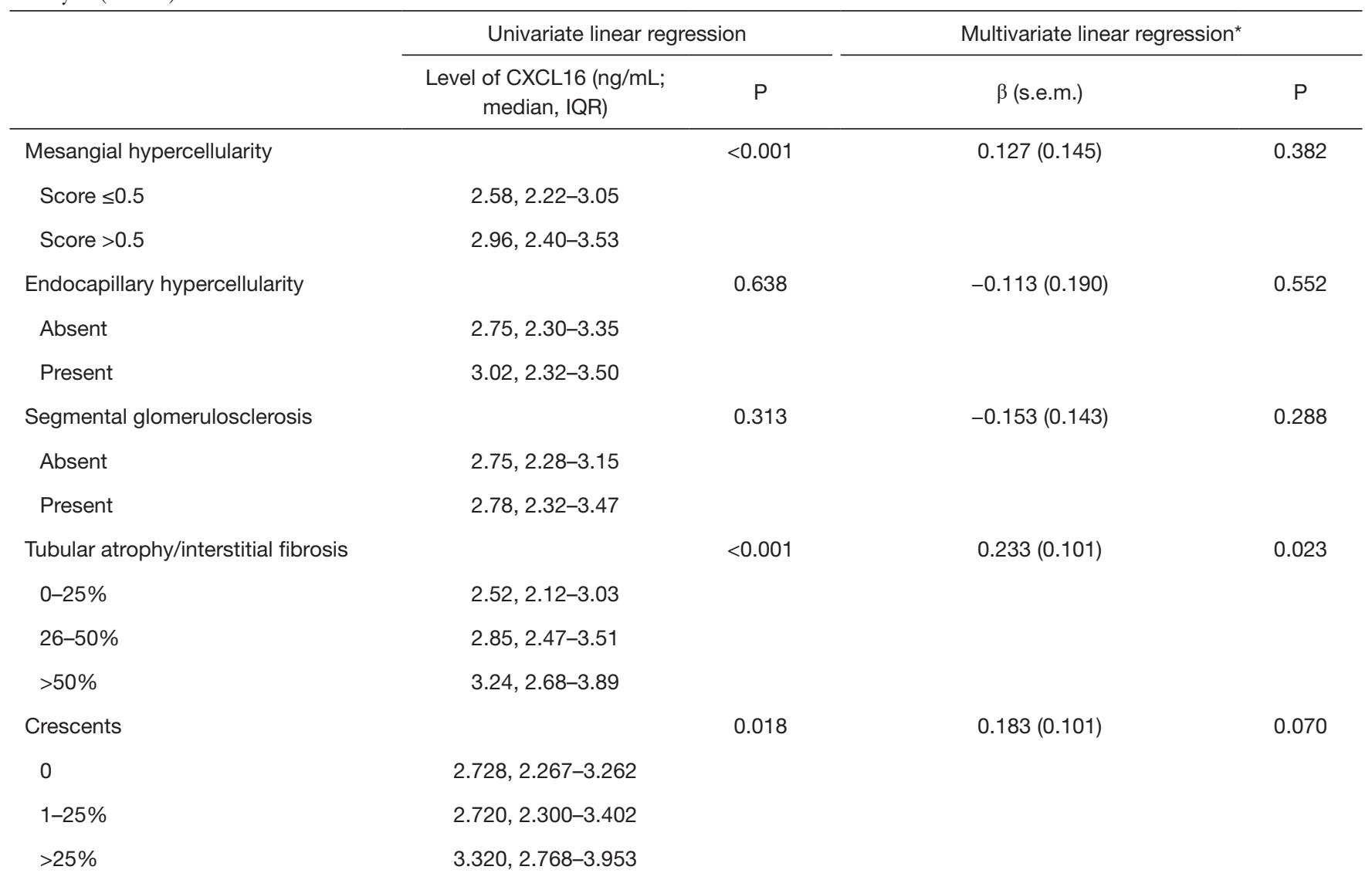

*Multivariate model: multivariate with the 5 pathologic features (mesangial hypercellularity, endocapillary hypercellularity, segmental glomerulosclerosis, tubular atrophy/interstitial fibrosis, crescents) plus age, gender, mean arterial pressure, and 24-h urine protein.

plasma CXCL16 levels were positively associated with renal CXCL16 expression in tissues.

\section{Correlations between CXCL16 levels and inflammatory cell infiltration}

CXCL16 acts as a chemoattractant molecule and recruits inflammatory cells to sites of inflammation. Therefore, we analyzed the association in IgAN patients of the infiltration of several inflammatory cells in kidney biopsy specimens with CXCL16 plasma levels. IHC was performed to quantify different subsets of leukocytes, including $\mathrm{CD}^{+} \mathrm{T}$ cells, $\mathrm{CD} 8^{+} \mathrm{T}$ cells, CD20 ${ }^{+} \mathrm{B}$ cells, and $\mathrm{CD} 68^{+}$ macrophages. We found an observable positive association between the infiltration frequency of $\mathrm{CD} 8^{+} \mathrm{T}$ cells and $\mathrm{CD}^{+} \mathrm{T}$ cells in clinical specimens and the plasma CXCL16 levels $(\mathrm{r}=0.301, \mathrm{P}=0.007 ; \mathrm{r}=0.237, \mathrm{P}=0.035$; Figure $3 A, B)$.
The plasma CXCL16 levels were also significantly correlated with the number of renal $\mathrm{CD} 20^{+} \mathrm{B}$ cell infiltration in IgAN patients ( $\mathrm{r}=0.317, \mathrm{P}=0.005$; Figure 3 C). No significant association was seen between the amount of $\mathrm{CD} 68^{+}$macrophage infiltration in clinical specimens and the plasma CXCL16 levels in our patients with IgAN $(\mathrm{r}=-0.020, \mathrm{P}=0.864$; Figure $3 D)$. Altogether, these results prove that plasma CXCL16 levels were correlated with the infiltration of inflammatory cells in renal tissues.

\section{Correlations between CXCL16 levels and outcome}

Seventeen events of the combined outcome (50\% reduction in eGFR or ESRD) occurred in a mean follow-up period of 41.3 months from the renal biopsy. Patients were divided by plasma CXCL16, and a high level of CXCL16 was associated with a reduction in the combined renal survival 

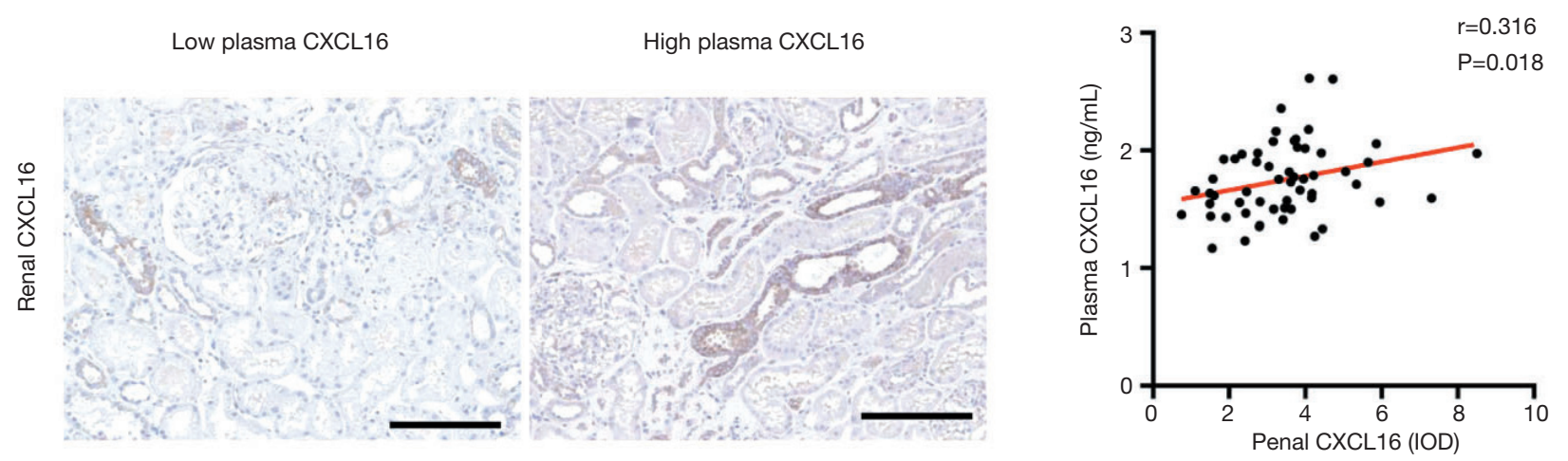

Figure 2 The renal IHC staining of CXCL16 in high and low levels of plasma CXCL16 (magnification $\times 200$, scale bar $=80 \mu \mathrm{m}$ ).

rate $(\mathrm{P}=0.001)$ (Figure 4). In Cox regression analysis, a high level of plasma CXCL16 (HR, 1.873; 95\% CI, 1.012-3.468, $\mathrm{P}=0.046)$ was an independent predictor for IgAN patients, along with age, gender, mean arterial pressure and $24 \mathrm{~h}$ Urine protein (Table 4). Strikingly, these results suggest that plasma CXCL16 levels were correlated with the progression of IgAN patients and may serve as a noninvasive biomarker of renal disease.

\section{Correlations between CXCL16 polymorphisms and plasma CXCL16 levels}

Finally, we analyzed the associations between 9 singlenucleotide polymorphisms of CXCL16 (rs2304973, rs2250333, rs1050998, rs3744700, rs2234358, rs1876444, rs8123, rs2277680, and rs1051009) and the plasma CXCL16 levels in IgAN patients. The 9 gene variants were tested in 222 patients in our cohort. However, we did not find any statistically significant association of these polymorphisms with the plasma CXCL16 levels in our IgAN cohort $(\mathrm{P}>0.05)$. Moreover, no significant association was seen between the 11 gene locus variants of CXCL16 and any clinical and pathological parameters of our IgAN patients. The details are shown in Table 5 .

\section{Discussion and conclusions}

IgAN is the most widespread primary chronic glomerular disease worldwide. However, the requirement of a renal biopsy for diagnosis impedes a description of the full consequences of this disease (2). Several recent studies have suggested that chemokine is involved in the development and progression of kidney diseases (6). We here investigated the plasma level of chemokine CXCL16, using a well- defined single central IgA cohort. This study is the first prospective clinical study to assess the prognostic significance of plasma CXCL16 on renal survival in a of long-term follow-up of IgAN patients. It could serve as a useful laboratory test to detect IgAN renal function and prognosis in the future, which could limit the need for invasive renal biopsies in such a patient population.

In previous research, serum CXCL16 was correlated with clinical and pathological parameters in diseases, such as lupus nephritis (15) and diabetic kidney disease (18). Our study also showed that the levels of plasma CXCL16 in our IgAN patient population were associated with the markers of renal function eGFR, serum creatinine (Scr), uric acid (UA), and blood urea nitrogen (BUN) and 24-hour protein levels. In multivariate linear regression analysis, UA and eGFR remained independently associated with plasma CXCL16. Crucially different from earlier research, our study focused on the relationship between circulating CXCL16 and IgAN renal pathology. The study revealed that in univariate analysis plasma CXCL16 levels were higher in patients with mesangial hypercellularity, tubular atrophy/interstitial fibrosis or more renal crescent proportion.

Furthermore, there was still a significant association between the tubular atrophy/interstitial fibrosis and plasma CXCL16 levels even after adjustment for clinical predictor variables by multivariate analysis. The mesangial hypercellularity and tubular atrophy/interstitial fibrosis lesions of the Oxford Classification have been reported to independently predict poor survival in large validation studies (22). Indeed, $\mathrm{Hu}$ et al. found that inflammationactivated CXCL16 pathway contributes to tubulointerstitial injury in mouse diabetic nephropathy (23), which further confirmed our results. However, our results did not find plasma CXCL16 independently associated with mesangial 
A

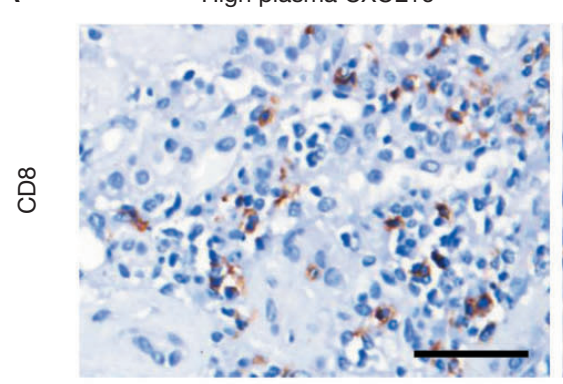

B

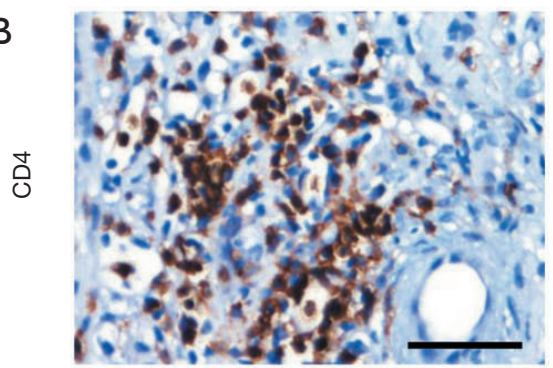

C

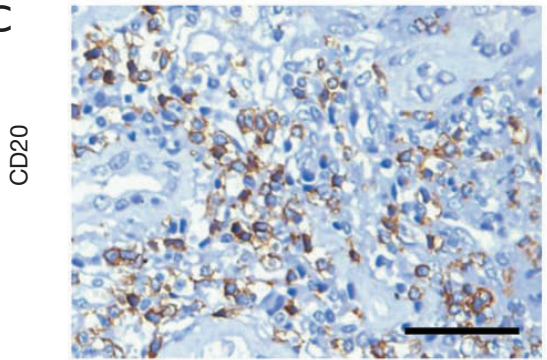

D

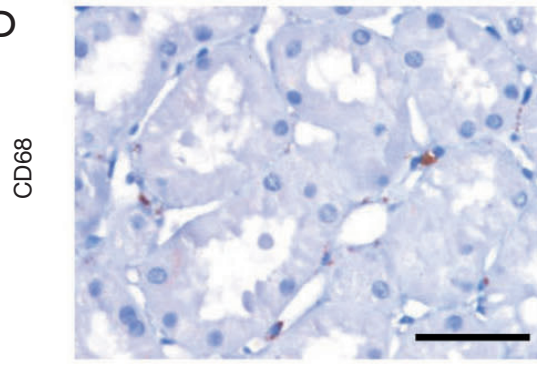

High plasma CXCL16
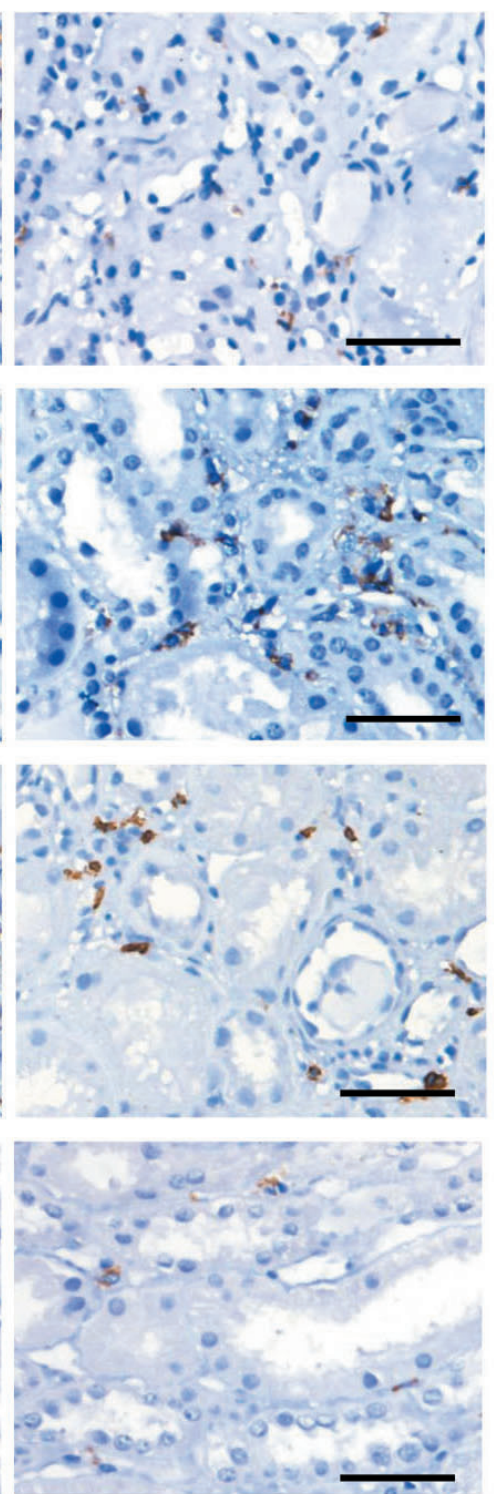
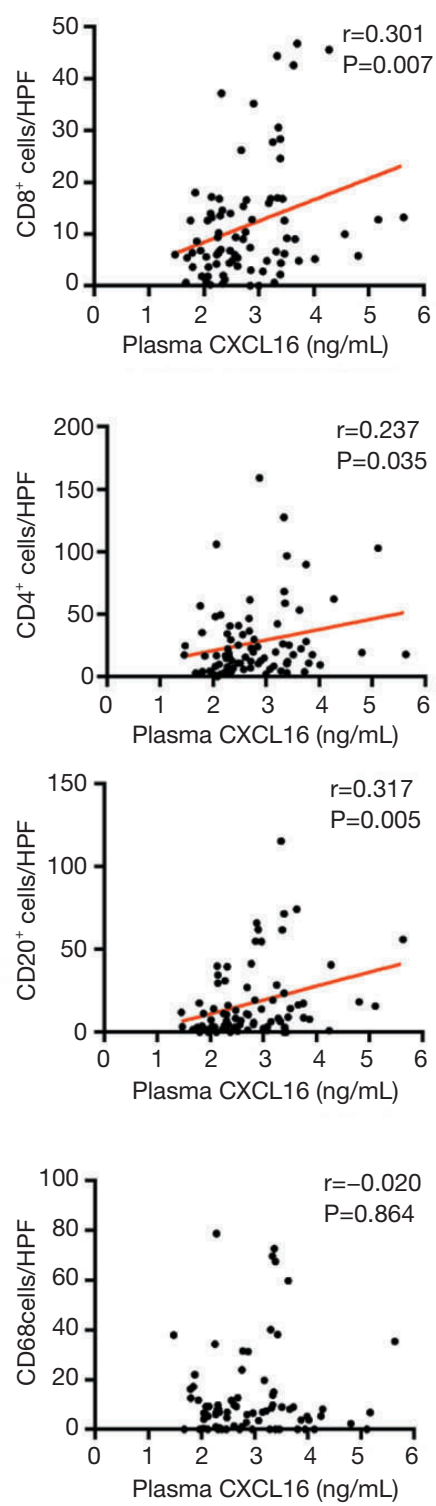

Figure 3 The IHC staining of CD8 ${ }^{+} \mathrm{T}$ cells (A), $\mathrm{CD} 4^{+} \mathrm{T}$ cells (B), CD20 $\mathrm{B}$ cells (C) and $\mathrm{CD} 68^{+}$macrophage (D) in high and low levels of plasma CXCL16 (magnification $\times 400$, scale bar $=40 \mu \mathrm{m})$.

hypercellularity in multivariate analysis, which may be affected by other factors. These results still demonstrated that plasma CXCL16 levels were positively associated with renal function and pathology.

Research has shown that the infiltration of inflammatory cells in renal tissue has prognostic significance in $\operatorname{IgAN}(7)$, but it has remained unclear whether plasma CXCL16 levels could reflect organ inflammation conditions. Our study analysis showed that plasma CXCL16 levels were significantly correlated with the infiltration of $\mathrm{CD}^{+} \mathrm{T}$ cells, $\mathrm{CD}^{+} \mathrm{T}$ cells and $\mathrm{CD} 20^{+} \mathrm{B}$ cells in the renal specimens, but not related to the infiltration of $\mathrm{CD} 68^{+}$macrophage cells in renal tissue. CXCR6 was mainly expressed in T cells (12). In vivo study demonstrated that CXCL16/CXCR6 takes part in T cell migration into the inflammation tissue $(14,24)$. We are first to show that plasma CXCL16 could reflect renal inflammatory cell infiltration. These results were consistent with CXCL16-mediated T cells accumulated in 
patients with vitiligo and atherosclerosis $(11,25)$.

Circulating CXCL16 levels have been associated with poor outcomes in various diseases, such as prostate cancer (26), cardiomyopathy (27), and acute ischemic stroke (28). Consistent with the above studies, we found that there was an independent effect of plasma CXCL16 levels on adverse outcomes during 41.3 months of follow-up. In addition, after adjusting for the confounding effects of age, gender, MAP, and 24-hour urine protein, the relationship of plasma CXCL16 and IgAN prognosis remained significant in multivariate Cox regression analysis. These results suggest that plasma CXCL16 levels might have a potent ability for renal outcome prognosis in IgAN.

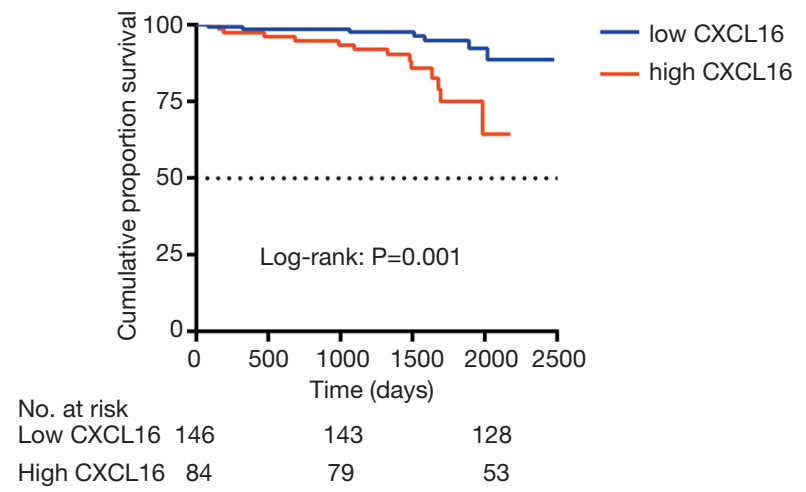

Figure 4 Kaplan-Meier curve of overall patient survival according to plasma CXCL16 level category. Patient survival was significantly better for the low CXCL16 group than for the high CXCL16 group (log-rank test, $\mathrm{P}=0.005)$.
There has been extensive study on the interaction of CXCL16 gene locus polymorphisms with multiple inflammatory diseases $(16,29)$. However, the effect of CXCL16 SNP on renal disease is still unknown. Our study is the first to investigate the association between polymorphisms of the CXCL16 gene and plasma CXCL16, along with the severity of renal function and renal outcomes in IgAN patients. Meanwhile, in our cohort study, the gene polymorphisms of these 9 CXCL16 genes did not display any association with the plasma CXCL16 levels, or with the severity of renal function and renal outcomes. We presumed that plasma CXCL16 levels were associated with the severity of diseases rather than genetic polymorphism, and the levels of plasma CXCL16, not CXCL16 polymorphism, play a key role in the renal outcome of $\operatorname{IgA}$ nephropathy.

The present study had several limitations that should be briefly acknowledged. First, the plasma CXCL16 levels were only assessed at baseline, and the variation in levels over time and treatment response should be further evaluated. Second, the sample size of the study was relatively small, and a prospective study is required to confirm the clinical utility of CXCL16 in IgAN patients further.

In summary, we demonstrated that the plasma CXCL16 levels were highly associated with the renal function, pathological damage, renal CXCL16 expression, and inflammatory cell infiltration in the renal tissue of $\operatorname{IgAN}$ patients. Furthermore, we were first to discover that that patients with heightened plasma CXCL16 levels were associated with a poor renal outcome in IgAN patients. Hence, plasma CXCL16 might be a non-invasive predictor for IgAN patients.

Table 4 Association between plasma CXCL16 levels and renal outcome in IgAN patients

\begin{tabular}{lccc}
\hline & & Multivariate Cox regression analysis \\
\cline { 2 - 4 } & HR & $95 \% \mathrm{Cl}$ & $\mathrm{P}$ value \\
\hline Age & 1.029 & $0.971-1.090$ & 0.333 \\
Gender & 0.537 & $0.189-1.530$ & 0.244 \\
MAP (mmHg) & 1.002 & $0.967-1.039$ & 0.898 \\
24-h Urine protein & 1.188 & $1.032-1.367$ & 0.016 \\
CXCL16 $(\mathrm{ng} / \mathrm{mL})$ & 1.873 & $1.012-3.468$ & 0.046 \\
\hline
\end{tabular}

HR, hazard ratio; 95\% Cl, 95\% confidence interval. Renal outcome was defined as a 50\% reduction in eGFR and ESRD. 
Table 5 Associations between CXCL16 polymorphisms and clinical parameters

\begin{tabular}{|c|c|c|c|c|c|c|c|}
\hline Polymorphism & Genotype & $\mathrm{N}$ & CXCL16, ng/mL & Scr, umol/L & eGFR, $\mathrm{ml} / \mathrm{min}$ per $1.73 \mathrm{~m}^{2}$ & 24-h urine protein, $\mathrm{g} / \mathrm{d}$ & Combine-event \\
\hline rs2304973 & CT & 30 & $2.987 \pm 0.787$ & $100.60 \pm 40.39$ & $84.40 \pm 35.75$ & $1.612 \pm 1.620$ & $3(10.0 \%)$ \\
\hline \multirow[t]{2}{*}{ rs2250333 } & $\mathrm{CC}$ & 109 & $2.806 \pm 0.744$ & $93.97 \pm 39.27$ & $87.04 \pm 30.10$ & $1.475 \pm 2.183$ & $6(5.5 \%)$ \\
\hline & CT & 99 & $2.954 \pm 0.862$ & $94.01 \pm 55.46$ & $90.12 \pm 31.23$ & $1.549 \pm 1.831$ & $9(9.1 \%)$ \\
\hline \multirow[t]{3}{*}{ rs1050998 } & $\mathrm{CC}$ & 79 & $2.892 \pm 0.807$ & $96.75 \pm 41.96$ & $84.98 \pm 30.08$ & $1.812 \pm 2.540$ & $5(6.3 \%)$ \\
\hline & CT & 107 & $2.856 \pm 0.839$ & $92.79 \pm 53.59$ & $91.73 \pm 31.44$ & $1.429 \pm 1.728$ & $10(9.3 \%)$ \\
\hline & $\pi$ & 35 & $2.960 \pm 0.774$ & $88.34 \pm 38.56$ & $90.01 \pm 29.54$ & $1.226 \pm 1.608$ & $1(2.9 \%)$ \\
\hline rs3744700 & GG & 174 & $2.927 \pm 0.855$ & $94.43 \pm 50.20$ & $89.05 \pm 30.94$ & $1.694 \pm 2.205$ & $13(7.5 \%)$ \\
\hline \multirow[t]{3}{*}{ rs2234358 } & GG & 97 & $2.909 \pm 0.752$ & $94.27 \pm 47.50$ & $88.49 \pm 31.16$ & $1.323 \pm 1.494$ & $9(9.3 \%)$ \\
\hline & GT & 99 & $2.921 \pm 0.927$ & $97.25 \pm 50.62$ & $86.24 \pm 30.84$ & $1.642 \pm 2.339$ & $5(5.1 \%)$ \\
\hline & $\pi$ & 25 & $2.650 \pm 0.511$ & $75.68 \pm 26.68$ & $102.30 \pm 25.41$ & $1.830 \pm 2.422$ & $2(8.0 \%)$ \\
\hline \multirow[t]{3}{*}{ rs1876444 } & $\mathrm{CC}$ & 80 & $2.899 \pm 0.805$ & $96.40 \pm 41.81$ & $85.39 \pm 30.11$ & $1.790 \pm 2.525$ & $5(6.3 \%)$ \\
\hline & $\mathrm{CT}$ & 108 & $2.871 \pm 0.862$ & $93.18 \pm 53.33$ & $90.95 \pm 31.48$ & $1.448 \pm 1.727$ & $10(9.3 \%)$ \\
\hline & $\pi$ & 34 & $2.964 \pm 0.786$ & $88.35 \pm 39.14$ & $90.49 \pm 29.84$ & $1.226 \pm 1.608$ & $1(2.9 \%)$ \\
\hline \multirow[t]{2}{*}{ rs8123 } & AA & 98 & $2.794 \pm 0.746$ & $95.58 \pm 41.21$ & $86.03 \pm 29.63$ & $1.629 \pm 2.289$ & $8(8.1 \%)$ \\
\hline & $A C$ & 108 & $2.954 \pm 0.889$ & $91.77 \pm 52.79$ & $91.77 \pm 31.20$ & $1.455 \pm 1.784$ & $7(6.5 \%)$ \\
\hline \multirow{2}{*}{ rs1051009 } & CT & 121 & $2.913 \pm 0.871$ & $91.22 \pm 43.56$ & $90.16 \pm 30.06$ & $1.363 \pm 1.614$ & $10(8.3 \%)$ \\
\hline & $\pi$ & 58 & $2.806 \pm 0.775$ & $95.57 \pm 43.63$ & $85.55 \pm 29.91$ & $2.018 \pm 2.881$ & $2(3.4 \%)$ \\
\hline
\end{tabular}

Values are expressed as mean \pm standard deviation, or number (percentage).

\section{Acknowledgments}

Funding: This work was financially supported by the International (regional) Cooperation and Exchange Projects, (NSFC-DFG, No. 81761138041), the National Natural Science Foundation of China (No. 81570667, 81470948, and 81670633); the Major Research Plan of the National Natural Science Foundation of China (No. 91742204), The National Key R\&D Program of China (No. 2018YFC1314003-1 and 2015BAI12B07), and
National Key Research and Development program (No. 2016YFC0906103).

\section{Footnote}

Conflicts of Interest: The authors have no conflicts of interest to declare.

Ethical Statement: The authors are accountable for all aspects of the work in ensuring that questions related 
to the accuracy or integrity of any part of the work are appropriately investigated and resolved. All the patients gave informed consent to participate in this study, and the study protocol was approved by the Ethical Committee of Huazhong University of Science and Technology (No. TJIRB20181108). The study was conducted in adherence to the Declaration of Helsinki.

Open Access Statement: This is an Open Access article distributed in accordance with the Creative Commons Attribution-NonCommercial-NoDerivs 4.0 International License (CC BY-NC-ND 4.0), which permits the noncommercial replication and distribution of the article with the strict proviso that no changes or edits are made and the original work is properly cited (including links to both the formal publication through the relevant DOI and the license). See: https://creativecommons.org/licenses/by-nc-nd/4.0/.

\section{References}

1. Lai KN, Tang SC, Schena FP, et al. IgA nephropathy. Nat Rev Dis Primers 2016;2:16001.

2. Wyatt RJ, Julian BA. IgA nephropathy. N Engl J Med 2013;368:2402-14.

3. Eardley KS, Kubal C, Zehnder D, et al. The role of capillary density, macrophage infiltration and interstitial scarring in the pathogenesis of human chronic kidney disease. Kidney Int 2008;74:495-504.

4. Myllymaki JM, Honkanen TT, Syrjanen JT, et al. Severity of tubulointerstitial inflammation and prognosis in immunoglobulin A nephropathy. Kidney Int 2007;71:343-8.

5. van Es LA, de Heer E, Vleming LJ, et al. GMP17-positive T-lymphocytes in renal tubules predict progression in early stages of IgA nephropathy. Kidney Int 2008;73:1426-33.

6. Pei G, Zeng R, Han M, et al. Renal interstitial infiltration and tertiary lymphoid organ neogenesis in IgA nephropathy. Clin J Am Soc Nephrol 2014;9:255-64.

7. Wang $\mathrm{Y}, \mathrm{Hu} \mathrm{Z}, \mathrm{Wu} \mathrm{J}$, et al. High renal DC-SIGN(+) cell density is associated with severe renal lesions and poor prognosis in patients with Immunoglobulin A Nephropathy. Histopathology 2019;74:744-58.

8. Weninger W, Biro M, Jain R. Leukocyte migration in the interstitial space of non-lymphoid organs. Nat Rev Immunol 2014;14:232-46.

9. Li CH, Xu LL, Zhao JX, et al. CXCL16 upregulates RANKL expression in rheumatoid arthritis synovial fibroblasts through the JAK2/STAT3 and p38/MAPK signaling pathway. Inflamm Res 2016;65:193-202.

10. Izquierdo MC, Martin-Cleary C, Fernandez-Fernandez $\mathrm{B}$, et al. CXCL16 in kidney and cardiovascular injury. Cytokine Growth Factor Rev 2014;25:317-25.

11. Li S, Zhu G, Yang Y, et al. Oxidative stress drives CD8+ T-cell skin trafficking in patients with vitiligo through CXCL16 upregulation by activating the unfolded protein response in keratinocytes. J Allergy Clin Immunol 2017;140:177-189.e9.

12. Scholz F, Schulte A, Adamski F, et al. Constitutive expression and regulated release of the transmembrane chemokine CXCL16 in human and murine skin. J Invest Dermatol 2007;127:1444-55.

13. Hofnagel O, Engel T, Severs NJ, et al. SR-PSOX at sites predisposed to atherosclerotic lesion formation mediates monocyte-endothelial cell adhesion. Atherosclerosis 2011;217:371-8.

14. Butcher MJ, Wu CI, Waseem T, et al. CXCR6 regulates the recruitment of pro-inflammatory IL-17A-producing T cells into atherosclerotic aortas. Int Immunol 2016;28:255-61.

15. Hassan AM, Farghal NMA, Hegab DS, et al. Serumsoluble CXCL16 in juvenile systemic lupus erythematosus: a promising predictor of disease severity and lupus nephritis. Clin Rheumatol 2018;37:3025-32.

16. Xu S, Cheng J, Cai MY, et al. The Impact of tagSNPs in CXCL16 Gene on the Risk of Myocardial Infarction in a Chinese Han Population. Dis Markers 2017;2017:9463272.

17. Brant SR, Okou DT, Simpson CL, et al. Genome-Wide Association Study Identifies African-Specific Susceptibility Loci in African Americans With Inflammatory Bowel Disease. Gastroenterology 2017;152:206-17.e2.

18. Elewa U, Sanchez-Nino MD, Mahillo-Fernandez I, et al. Circulating CXCL16 in Diabetic Kidney Disease. Kidney Blood Press Res 2016;41:663-71.

19. Zhao L, Wu F, Jin L, et al. Serum CXCL16 as a novel marker of renal injury in type 2 diabetes mellitus. PLoS One 2014;9:e87786.

20. Garcia GE, Truong LD, Li P, et al. Inhibition of CXCL16 attenuates inflammatory and progressive phases of antiglomerular basement membrane antibody-associated glomerulonephritis. Am J Pathol 2007;170:1485-96.

21. Luo R, Guo SM, Li YQ, et al. Plasma fractalkine levels are associated with renal inflammation and outcomes in immunoglobulin A nephropathy. Nephrol Dial Transplant 2019;34:1549-58.

22. Coppo R, Troyanov S, Bellur S, et al. Validation of the Oxford classification of IgA nephropathy in cohorts 
with different presentations and treatments. Kidney Int 2014;86:828-36.

23. Hu ZB, Ma KL, Zhang Y, et al. Inflammation-activated CXCL16 pathway contributes to tubulointerstitial injury in mouse diabetic nephropathy. Acta Pharmacol Sin 2018;39:1022-33.

24. Riedel JH, Paust HJ, Turner JE, et al. Immature renal dendritic cells recruit regulatory CXCR6(+) invariant natural killer T cells to attenuate crescentic GN. J Am Soc Nephrol 2012;23:1987-2000.

25. Galkina E, Harry BL, Ludwig A, et al. CXCR6 promotes atherosclerosis by supporting T-cell homing, interferongamma production, and macrophage accumulation in the aortic wall. Circulation 2007;116:1801-11.

26. Richardsen E, Ness N, Melbo-Jorgensen C, et al. The

Cite this article as: Luo R, Yang Y, Cheng YC, Chang D, Liu TT, Li YQ, Dai W, Zuo MY, Xu YL, Zhang CX, Ge SW, Xu G. Plasma chemokine CXC motif-ligand 16 as a predictor of renal prognosis in immunoglobulin A nephropathy. Ann Transl Med 2020;8(6):381. doi: 10.21037/atm.2020.02.05 prognostic significance of CXCL16 and its receptor C-X-C chemokine receptor 6 in prostate cancer. Am J Pathol 2015;185:2722-30.

27. Borst O, Schaub M, Walker B, et al. CXCL16 is a novel diagnostic marker and predictor of mortality in inflammatory cardiomyopathy and heart failure. Int J Cardiol 2014;176:896-903.

28. Ueland T, Smedbakken LM, Hallen J, et al. Soluble CXCL16 and long-term outcome in acute ischemic stroke. Atherosclerosis 2012;220:244-9.

29. Chua KH, Ng JG, Ng CC, et al. Association of NOD1, CXCL16, STAT6 and TLR4 gene polymorphisms with Malaysian patients with Crohn's disease. PeerJ 2016;4:e1843. 